\title{
PREGUNTA DE INVESTIGACIÓN Y ESTRATEGIA PICOT
}

\author{
RESEARCH QUESTION AND PICOT STRATEGY
}

PERGUNTA DE PESQUISA EESTRATÉGIA PICOT

\section{CARLOS PEÑAHERRERA OVIEDO', JAIME SORIA VITERI ${ }^{1}$}

${ }^{1}$ Universidad Católica de Santiago de Guayaquil, Guayaquil, Ecuador

\section{Resumen}

Cuando se observa un problema en la atención de la salud, y se desea encontrar la forma de solucionarlo, se origina una pregunta de investigación. Es esta interrogante la que dicta posteriormente cómo se llevará a cabo la investigación, qué diseño de estudio es el más adecuado para responderla y qué resultados se espera obtener. Por este motivo, es fundamental que todo investigador esté familiarizado con las estrategias para elaborar una pregunta de investigación, pues cada uno de sus componentes debe definirse. En la presente revisión se realizará una descripción sobre las características de una buena pregunta de investigación y su construcción mediante la estrategia conocida como PICOT. Además, se brinda una descripción de los posibles diseños epidemiológicos que pueden derivarse de esta pregunta, sus ventajas y desventajas. Esta información será importante para la correcta aplicación de la medicina basada en evidencia.

PALABRAS CLAVE: investigación operativa, diseños de investigaciones epidemiológicas, medicina basada en la evidencia.

Abstract

When a problem in health care is observed and a way to solve it is needed, a research question originates. It is this question which then dictates how research will be conducted, what study design is best suited to answer it and what results are expected. For this reason, it is essential that every researcher is familiarized with strategies to develop a research question, as each one of its components must be defined. In this review a description of the characteristics of a good research question and its construction by the strategy known as PICOT will be made. In addition, this paper provides a description of possible epidemiological designs that can be derived from this question, its advantages and disadvantages. This information will be important for the correct application of evidence-based medicine.

KEYWORDS: operations research, epidemiologic research design, evidence-based medicine.

Resumo

Quando há um problema em matéria de cuidados de saúde e se deseja encontrar a maneira de resolvê-lo, origina-se uma pergunta de pesquisa. É esta questão que dita posteriormente como efetuar a pesquisa, que desenho de estudo é o mais adequado para responder e quais resultados espera se obtiver. Por este motivo, é fundamental que qualquer pesquisador esteja familiarizado com as estratégias para desenvolver uma pergunta de pesquisa, porque cada um de seus componentes deve ser definido. Na presente revisão será efetuada uma descrição das características de uma boa pergunta de pesquisa e sua construção pela estratégia conhecida como o PICOT. Além disso, fornece-se uma descrição dos possíveis modelos epidemiológicos que podem ser derivados de esta questão e de suas vantagens e desvantagens. Esta informação será importante para a correta aplicação da medicina baseada em evidência.

PALABRAS-CHAVE: pesquisa operacional, projetos de pesquisa epidemiológica, medicina baseada em evidências. 
INTRODUCCIÓN

La investigación clínica está orientada a resolver dudas con el objetivo de obtener nuevos conocimientos, por lo que a continuación se presentará un análisis sobre la pregunta de investigación y cómo construirla. Los médicos se plantean entre 0,7 y 18,5 preguntas por cada 10 pacientes que atienden, de las cuales muchas quedan sin resolver y otras llevan al desarrollo de una investigación. ${ }^{1}$ El principal objetivo de la investigación científica es encontrar respuesta a la pregunta planteada, con el fin de compartir la información con el resto de los profesionales en salud, ${ }^{2-4}$ mediante publicaciones, artículos científicos, textos, etc.

La pregunta de investigación es el motivo de toda publicación. De ella depende el diseño de la investigación, la población que debe ser seleccionada, el abordaje de la discusión, la consulta bibliográfica sobre el tema, etc. Define las características que constituyen el artículo investigativo y como objetivo del mismo está una respuesta a dicha pregunta. ${ }^{2,5,6}$ Por esto, es imperativo que aquellos que deseen iniciar una investigación con miras a la publicación de los resultados, estén familiarizados con la completa estructuración de la pregunta de investigación.

LA PREGUNTA DE INVESTIGACIÓN

Una investigación necesita sostenerse en base a una pregunta concisa. Los problemas clínicos que surgen durante la práctica diaria originan preguntas que llevan al desarrollo de un proyecto investigativo. ${ }^{7}$ En la práctica de la medicina basada en evidencia (tabla 1), la formulación de la pregunta aparece muy temprano en el proceso, sólo después de la identificación de un problema. ${ }^{8}$ La pregunta no debe confundirse con el título de la investigación; el título trata de resumir e introducir al proyecto, motivo por el que suele originarse la pregunta de investigación. ${ }^{3}$ Además la pregunta debe ser clara, tanto que debería poder ser identificada fácilmente por toda persona que lea el artículo. ${ }^{6}$

\section{TABLA 1. ETAPAS DE LA MEDICINA BASADA EN EVIDENCIA ${ }^{8}$}

\begin{tabular}{l}
\hline 1. Identificación de un problema clínico. \\
\hline 2. \\
3. Burmulación de una pregunta de investigación relevante y específica. \\
4. Evidencia científica. \\
5. Evaluación de la evidencia encontrada. \\
6. Implementación de la evidencia en la atención de pacientes. \\
7. Evaluación de los resultados obtenidos. \\
\hline
\end{tabular}

El tipo de investigación y el diseño del estudio se derivan del tipo de pregunta que se plantea el autor y sobre lo que se busca responder. ${ }^{6}$ Así, si tenemos una pregunta relacionada al diagnóstico de una enfermedad, se realizará un estudio de cohorte o transversal; mientras que si la pregunta es sobre etiología lo mejor será un estudio de casos y controles o de cohorte; este último también resolverá preguntas sobre pronóstico clínico. Finalmente, en caso de tener una pregunta sobre tratamiento, vamos a construir un ensayo clínico controlado y aleatorizado. ${ }^{4,9}$ No se puede decidir un diseño investigativo sin haber formulado primero la pregunta, pues ésta podría cambiar la intención inicial del autor sobre el tipo de estudio a realizar. Una buena pregunta debe cumplir ciertos requisitos para determinar su utilidad y el grado de aporte científico que brindará. Para esto, se utilizan los criterios FINER (tabla 2). 5,6 Si la pregunta cumple con estos requisitos, se puede dar paso a la investigación. Nuevamente, la pregunta debe estar correctamente estructurada, para luego poder evaluarla según estos criterios. ${ }^{10}$

TABLA 2. CRITERIOS FINER PARA EVALUAR LAS PROPIEDADES DE UNA PREGUNTA DE INVESTIGACIÓN6

\begin{tabular}{|ll}
\hline COMPONENTE & ESTRATEGIAS PARA EVALUAR \\
\hline ¿Es factible la investigación? & $\begin{array}{l}\text { Realizar un estudio piloto, modificar } \\
\text { criterios de inclusión, conseguir } \\
\text { colaboradores y opiniones de } \\
\text { expertos, utilizar diseños } \\
\text { menos costosos, buscar resultados } \\
\text { comunes }\end{array}$ \\
¿Es interesante? & $\begin{array}{l}\text { Analizar si la investigación interesa } \\
\text { al investigador, a los colaboradores, } \\
\text { a los financiadores }\end{array}$ \\
\hline ¿Es novedosa? & $\begin{array}{l}\text { Conocer la literatura, obtener la } \\
\text { guía de un experto en investigación } \\
\text { ¿ un mentor }\end{array}$ \\
\hline ¿Es una investigación ética? & $\begin{array}{l}\text { Estar familiarizado con la } \\
\text { declaración de Helsinki, Buenas } \\
\text { Prácticas Clínicas (GCP), etc., } \\
\text { obtener aprobación de Comité de }\end{array}$ \\
Ética
\end{tabular}

Existen formas distintas de construir una pregunta de investigación. Una forma es utilizando las proposiciones: quién, cuándo, dónde, qué, cómo y por qué. Estos componentes deben estar correctamente definidos y reconocidos en la pregunta. ${ }^{5}$ Por ejemplo: “¿Por qué una parte 
de los pacientes con diabétes en la ciudad de Guayaquil no se adhieren al tratamiento con hipoglicemiantes orales en los primeros años del diagnóstico?”. Otro método es el propuesto por Bordage y Dawson, ${ }^{11}$ que consta de ocho pasos y 28 preguntas, de los cuales el primer paso (y sus correspondientes nueve preguntas) se enfoca exclusivamente en la construcción de la pregunta de investigación. Por último, tenemos la estrategia PICOT, un método que es a la vez completo y simple de seguir. ${ }^{2}$

\section{LA ESTRATEGIA PICOT PARA CONSTRUIR PREGUNTAS DE} INVESTIGACIÓN

Descrito por primera vez por Richardson y cols. en 1995, ${ }^{12}$ el acrónimo PICO (al que luego se le agregó la letra $\mathrm{T}$ para establecerlo como PICOT), es ampliamente recomendado para la elaboración de la pregunta de investigación. ${ }^{6}$ El uso rutinario de esta estrategia permite que la construcción contenga todos los componentes necesarios para establecer las características del estudio. ${ }^{8}$ La estrategia PICOT ayuda a establecer el tipo de evidencia necesaria para resolverla, e incluso a mejorar la búsqueda de información de bases de datos como PubMed. ${ }^{1,8,13}$ Los componentes básicos de la pregunta según la estrategia PICOT se muestran en la tabla 3.

\section{TABLA 3. COMPONENTES DEL ACRÓNIMO PICOT2,4-6,8,9}

\begin{tabular}{|c|c|c|}
\hline$P$ & $\begin{array}{l}\text { Población o } \\
\text { problema de } \\
\text { interés }\end{array}$ & $\begin{array}{l}\text { Puede ser una población o un solo paciente, } 0 \text { un } \\
\text { problema de salud. En el caso de poblaciones, } \\
\text { los participantes suelen compartir características } \\
\text { generales. }\end{array}$ \\
\hline I & Intervención & $\begin{array}{l}\text { Es la condición que se va a estudiar. Puede ser un } \\
\text { tratamiento administrado, una medida de prevención, } \\
\text { un método diagnóstico, una enfermedad, un factor de } \\
\text { riesgo. }\end{array}$ \\
\hline C & Comparación & $\begin{array}{l}\text { El grupo con el que se compara a la intervención, no } \\
\text { presenta la condición estudiada. Ejemplos: pacientes } \\
\text { que reciben tratamiento estándar o ningún tratamiento, } \\
\text { un grupo de controles sanos, individuos no expuestos a } \\
\text { un factor de riesgo. }\end{array}$ \\
\hline 0 & $\begin{array}{l}\text { Outcome } \\
\text { (resultado) }\end{array}$ & $\begin{array}{l}\text { Es el resultado que se espera obtener de la investiga- } \\
\text { ción, tanto en la Intervención como en su comparación. }\end{array}$ \\
\hline $\mathrm{T}$ & $\begin{array}{l}\text { Tiempo o tipo } \\
\text { de estudio }\end{array}$ & $\begin{array}{l}\text { Describe el marco de tiempo en el que se realiza la in- } \\
\text { vestigación, o el tiempo esperado para la aparición del } \\
\text { resultado. Los diferentes tipos de estudio (transversal, } \\
\text { cohorte, ensayo clínico, etc.) se ajustan al marco } \\
\text { de tiempo necesario para encontrar el resultado. No } \\
\text { siempre es requerido, pero es recomendable. }\end{array}$ \\
\hline
\end{tabular}

La mejor forma de aprender la forma de construir preguntas de investigación es con un ejemplo que muestre el proceso de formulación desde el inicio. Supongamos que en un servicio hospitalario se observa una incidencia alta de embolismo cerebral en pacientes con fibrilación auricular a pesar del tratamiento con warfarina, y los médicos desean empezar a evaluar otro tratamiento, como el dabigatrán, para intentar obtener mejores resultados. La pregunta inicialmente surge como "¿Dabigatrán es mejor que warfarina en la fibrilación auricular?". Falta detalle en esta pregunta inicial, pues no se ha definido adecuadamente todos los componentes en esta pregunta. Por lo tanto, el diseño del estudio y la búsqueda inicial de bibliografía pueden resultar poco eficiente. Si, por otro lado, se establece primero cada componente del PICOT para luego construir la pregunta, como se observa en la figura, obtendremos una pregunta completa y detallada que nos lleve a una metodología adecuada del estudio a realizar.

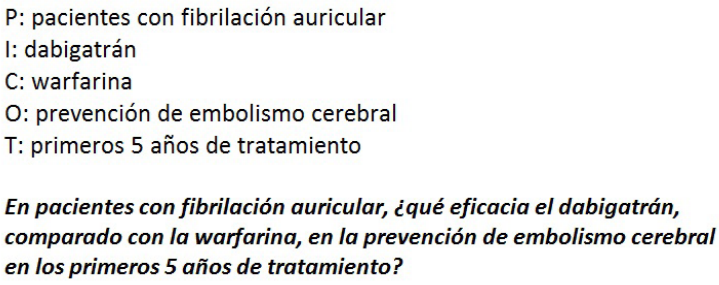

Figura. Ejemplo de la construcción adecuada de una pregunta de investigación utilizando el sistema PICOT. Peñaherrera, 2015.

El ejemplo mostrado plantea una pregunta sobre tratamiento, por lo cual el tipo de estudio o diseño recomendado será un ensayo clínico controlado. La eficiencia de la utilización de la estrategia PICOT para elaborar la pregunta que da inicio de los estudios experimentales se ha demostrado en reportes que correlacionan el número de parámetros respetados del PICOT, con la calidad del artículo publicado. ${ }^{14-16}$ En el caso de los estudios observacionales, el componente "intervención" de la pregunta será el factor de riesgo o la enfermedad estudiada versus la "comparación" que será un grupo de individuos que no presenten esa característica. ${ }^{9,12}$ El acrónimo PICOT es ideal para construir preguntas sobre terapéutica, mientras que para diagnóstico, causalidad o pronóstico, podría ser necesario modificar sus componentes. ${ }^{1}$ Es común que ciertos estudios transversales que investigan prevalencia o métodos diagnósticos, no reporten un parámetro de comparación, pero es recomendable hacerlo para mejorar la calidad del manuscrito. 
CONCLUSIONES

Como se ha expresado, es vital la elaboración de una pregunta de investigación adecuadamente construida, como primer paso para el inicio de todo tipo de estudio. Una investigación no puede desarrollarse si no tenemos claro qué duda queremos responder. Así mismo, la pregunta nos orientará hacia el tipo de estudio que debemos realizar, el tipo de estudio o diseño y marco de tiempo en el que se lo realizará. La estrategia PICOT es una forma eficaz y sencilla de elaborar una buena pregunta de investigación e iniciar un proyecto debidamente fundamentado en una interrogante clara.

\section{REFERENCIAS BIBLIOGRÁFICAS}

1. Huang X, Lin J, Demner-Fushman D. Evaluation of PICO as a Knowledge Representation for Clinical Questions. AMIA Annu Symp Proc. 2006;2006:359-63.

2. Riva JJ, Malik KMP, Burnie SJ, Endicott AR, Busse JW. What is your research question? An introduction to the PICOT format for clinicians. J Can Chiropr Assoc. 2012 Sep;56(3):167-71.

3. Day RA. Como Escribir Y Publicar Trabajos Cientificos. 3rd ed. Washington D.C.: Pan American Health Org; 2005. $271 \mathrm{p}$.

4. Martín Rodero H, Galindo Villardón MP. Medicina Basada en la Evidencia y meta-análisis: una aproximación conceptual, histórica y metodológica [Internet] [Máster en Análisis Avanzado de Datos Multivariantes]. [Salamanca]: Universidad de Salamanca; 2011 [cited 2015 Jul 8]. Available from: http://gredos.usal.es/jspui/ handle/10366/123467

5. Martínez-González A, Sánchez-Mendiola M. La pregunta de investigación en educación médica. Investig En Educ Médica. 2015 Jan;4(13):42-9.
6. Thabane L, Thomas T, Ye C, Paul J. Posing the research question: not so simple. Can J Anaesth J Can Anesth. 2009 Jan;56(1):71-9.

7. Nobre MRC, Bernardo WM, Jatene FB. Evidence based clinical practice. Part 1: well structured clinical questions. Rev Assoc Médica Bras. 2003 Jan;49(4):445-9.

8. Santos CM da C, Pimenta CA de M, Nobre MRC. The PICO strategy for the research question construction and evidence search. Rev Lat Am Enfermagem. 2007 Jun;15(3):508-11.

9. Echevarria IM, Walker S. To make your case, start with a PICOT question. Nursing (Lond). 2014 Feb;44(2):18-9.

10. Fernández SP. Elementos básicos en el diseño de un estudio. Cad Aten Primaria. 1996;3(2):83-5.

11. Bordage G, Dawson B. Experimental study design and grant writing in eight steps and 28 questions. Med Educ. 2003 Apr;37(4):376-85.

12. Richardson WS, Wilson MC, Nishikawa J, Hayward RS. The well-built clinical question: a key to evidence-based decisions. ACP J Club. 1995 Dec;123(3):A12-3.

13. Schardt C, Adams MB, Owens T, Keitz S, Fontelo P. Utilization of the PICO framework to improve searching PubMed for clinical questions. BMC Med Inform Decis Mak. 2007 Jun 15;7:16.

14. Rios LP, Ye C, Thabane L. Association between framing of the research question using the PICOT format and reporting quality of randomized controlled trials. BMC Med Res Methodol. 2010 Feb 5;10(1):11.

15. Borg Debono V, Zhang S, Ye C, Paul J, Arya A, Hurlburt L, et al. A look at the potential association between PICOT framing of a research question and the quality of reporting of analgesia RCTs. BMC Anesthesiol. 2013;13(1):44.

16. Cruz Pareja E, García Santibáñez R, Soria Viteri J. Jerarquía de los diseños epidemiológicos. Revisión de las publicaciones de los 17 años de la. Rev Ecuat Neurol. 2009;18(1-2):111-6. 